\title{
The temperature-mass relation in magnetized galaxy clusters
}

\author{
K. Dolag ${ }^{1}$, A. Evrard ${ }^{2}$, and M. Bartelmann ${ }^{1}$ \\ 1 Max-Planck-Institut für Astrophysik, PO Box 1317, 85741 Garching, Germany \\ 2 Dept. of Physics, University of Michigan, Ann Arbor, MI 48109-1120, USA \\ Received 9 June 2000 / Accepted 9 January 2001
}

\begin{abstract}
We use cosmological, magneto-hydrodynamic simulations of galaxy clusters to quantify the dynamical importance of magnetic fields in these clusters. The set-up of initial magnetic field strengths at high redshifts is chosen such that observed Faraday-rotation measurements in low-redshift clusters are well reproduced in the simulations. We compute the radial profiles of the intracluster gas temperature and of the thermal and magnetic pressure in a set of clusters simulated in the framework of an Einstein-de Sitter and a low-density, spatially-flat CDM cosmological model. We find that, for a realistic range of initial magnetic field strengths, the temperature of the intracluster gas changes by less than $\approx 5 \%$
\end{abstract}

Key words. magnetic fields, galaxies: clusters: general

\section{Introduction}

Magnetic fields are common in galaxy clusters. They are inferred from Faraday-rotation measurements (Vallee et al. 1986, 1987) in polarised background radio sources, and from diffuse radio haloes around galaxy clusters (Kronberg 1994). Depending on several assumptions, most notably on the characteristic length scale of field coherence, magnetic field strengths inferred from observations in typical galaxy cluster centres range from $\approx 0.1 \mu \mathrm{G}$ up to some tens of $\mu \mathrm{G}$.

Provided the magnetic field is tangled on sufficiently small scales for it to be considered randomised, it contributes a non-thermal pressure $p_{\mathrm{B}}$, which is related to the thermal pressure $p_{\text {th }}$ through

$\frac{p_{\mathrm{B}}}{p_{\mathrm{th}}}=2.5 \%\left(\frac{|\boldsymbol{B}|}{3 \mu \mathrm{G}}\right)^{2}\left(\frac{n}{10^{-3} \mathrm{~cm}^{-3}}\right)^{-1}\left(\frac{T}{10^{8} \mathrm{~K}}\right)^{-1}$

Conversely, magnetic fields ordered on scales comparable to the cluster scale can add anisotropic non-thermal pressure.

The structure of intracluster magnetic fields is largely unclear at present, and consequently the magnetic field strengths in clusters are equally uncertain. We investigate in this paper the amount of non-thermal pressure support due to realistic magnetic fields in simulated galaxy clusters.

Our approach is as follows (see Dolag et al. 1999). We set up gas-dynamical cosmological cluster simulations at high redshift and impose magnetic fields of variable strength and structure as summarised in Table 2 below.

Send offprint requests to: K. Dolag,

e-mail: kdolag@ibm-2.mpa-garching.mpg.de
Since the origin of magnetic fields on cluster scales is unknown, we use two extreme types of seed fields, namely either completely homogeneous or chaotic initial magnetic field structures. The evolution of the intracluster fields is then computed during cluster collapse. The initial mean magnetic field strength (or energy density) is a free parameter in our simulations. We choose its range such that the simulated clusters at low redshift well reproduce the statistics of observed Faraday-rotation measurements taken from Kim et al. (1991). It turned out in earlier work (Dolag et al. 1999; hereafter DBL99) that (i) the initial field structure is irrelevant because the final field structure is completely determined by the dynamics of the cluster collapse, and (ii) initial field strengths of order $10^{-9} \mathrm{G}$ at the initial redshift of the simulations (see Table 1) are adequate to reproduce the measured Faradayrotation statistics.

\section{Models}

We use a set of 90 numerically simulated galaxy clusters for investigating the effect of magnetic fields on the temperature and pressure stratification in these clusters. Here, we summarise the numerical technique only briefly because details on the methods applied, their implementation, and their performance in test problems have been published elsewhere (DBL99).

\subsection{Numerical method: GrapeMSPH}

We use the cosmological MHD code described in DBL99 to simulate the formation of magnetised galaxy clusters from an initial density perturbation field. The code combines 
the merely gravitational interaction of a dark-matter component with the hydrodynamics of a gaseous component. The gravitational interaction of the particles is evaluated on GRAPE boards (cf. Sugimoto et al. 1990), while the gas dynamics is computed in the SPH approximation (Lucy 1977; Monaghan 1992). The original "GrapeSPH" code (Steinmetz 1996) was extended by adding the magnetohydrodynamic equations to trace the evolution of the magnetic fields. Because of the assumed ideal electric conductivity, the fields are frozen into the gas flow. The back-reaction of the magnetic field on the gas via the Lorentz force is included. The numerical viscosity required to capture shocks in SPH is chosen such that angularmomentum transport in presence of shear flows is carefully controlled. The SPH kernel width is automatically adapted to the local number density of SPH particles, which results in an adaptive spatial resolution of the code.

Extensive tests of the code were performed and described in DBL99. The code succeeds in solving the coplanar MHD Riemann problem posed by Brio \& Wu (1988). Although the simulated magnetic field is not strictly divergence-free, $\nabla \cdot \boldsymbol{B}$ is always negligible compared to the magnetic field divided by a typical length scale of $\boldsymbol{B}$ (see DBL99 for details). The code also assumes the intracluster medium to be an ideal gas with an adiabatic index of $\gamma=5 / 3$, and neglects cooling. The surroundings of the clusters are important because their tidal fields affect the overall cluster structure and the merger history of the clusters. Therefore, the cluster simulation volumes are surrounded by a layer of boundary particles whose purpose it is to accurately represent the tidal fields of the cluster neighbourhood. Here we specially focus on the dynamical influence of the magnetic field in galaxy clusters, e.g. on the pressure support and the temperature in the clusters.

\subsection{Initial conditions}

We set up cosmological initial conditions for two CDMdominated universes (EdS and FlatLow) as specified in Table 1. For each cosmology, we calculate ten different realisations of the initial density-fluctuation field at redshift $z_{\text {ini }}$, which result in clusters of different final masses and dynamical states at redshift $z=0$. We simulate each of these clusters with up to five different initial magnetic field configurations, listed in Table 2, yielding a total of 90 cluster models.

Table 1. Parameters of the EdS and the FlatLow models

\begin{tabular}{|l|cccccc|}
\hline model & $H_{0}$ & $\Omega_{0}$ & $\Lambda$ & $\sigma_{8}$ & $f_{\text {baryon }}$ & $z_{\text {ini }}$ \\
\hline EdS & 0.5 & 1.0 & 0.0 & 1.2 & $5 \%$ & 15 \\
FlatLow & 0.7 & 0.3 & 0.7 & 1.05 & $10 \%$ & 20 \\
\hline
\end{tabular}

In lack of any detailed knowledge on the origin of primordial seed fields, we explore two extreme cases of initial field configurations. In one case ("homogeneous"), we assume that the field is initially constant throughout the cluster volume. In the other case ("chaotic"), we let the initial field orientation vary randomly from place to place, subject only to the condition that $\nabla \cdot B=0$. The initial field strengths in both cases are determined by setting the mean field energy densities. Table 2 summarises the initial field set-ups and the mean field strengths in the cluster cores. The initial field strengths are of order $10^{-9} \mathrm{G}$, the final field strengths of order $\mu \mathrm{G}$.

Table 2. Initial magnetic fields (Col. 2) and the resulting final magnetic field strengths in the simulated clusters (Cols. 3 and 4 for EdS and FlatLow models, respectively). The final values are an average of the magnetic field over the central region (within a radius of $350 \mathrm{kpc}$ ) and over all ten clusters for each cosmology

\begin{tabular}{|l|c|c|c|}
\hline initial field & $B_{\text {ini }}$ & $\left\langle B_{\text {final }}\right\rangle_{\text {core }}^{\mathrm{EdS}}$ & $\left\langle B_{\text {final }}\right\rangle_{\text {core }}^{\text {FTatLow }}$ \\
\hline no & $0.0 \mathrm{G}$ & - & - \\
low (chaotic) & $0.210^{-9} \mathrm{G}$ & $0.4 \mu \mathrm{G}$ & - \\
low & $0.210^{-9} \mathrm{G}$ & $0.4 \mu \mathrm{G}$ & $0.3 \mu \mathrm{G}$ \\
medium & $1.010^{-9} \mathrm{G}$ & $1.1 \mu \mathrm{G}$ & $0.8 \mu \mathrm{G}$ \\
high & $5.010^{-9} \mathrm{G}$ & $2.5 \mu \mathrm{G}$ & $2.0 \mu \mathrm{G}$ \\
\hline
\end{tabular}

\subsection{Evolution of intra-cluster fields}

At low redshifts, our simulated clusters reach temperatures of $k T \lesssim 10 \mathrm{keV}$, in agreement with observed clusters of similar mass. The gas densities in their cores range within $10^{-4}-10^{-3} \mathrm{~cm}^{-3}$, as inferred from X-ray luminosities and temperatures of observed clusters.

In agreement with our earlier paper (DBL99), we find that the structure of the intra-cluster magnetic fields at low redshifts is entirely independent on the structure of the initial magnetic field. For homogeneous and chaotic initial field set-ups, the mean strength and the coherence length are the same. Initial fields of $10^{-9} \mathrm{G}$ at $z_{\text {ini }}$ turn into final fields of $\mu \mathrm{G}$ strength at redshift zero, and the typical coherence length of the fields is of order $50 \mathrm{kpc} / h$. In comparison to expectations based on magnetic flux conservation in spherically collapsing spheres, the amplification of the field is one order of magnitude higher because of shear flows in the intracluster plasma.

During cluster collapse, the magnetic field is highly compressed, twisted, tangled and sheared. Any information on its initial structure is lost in that process. This is confirmed by Fig. 1, where it is shown that the magnetic pressure support does not depend on the initial field structure. We can therefore restrict our study to the homogeneous initial field set-up, which is numerically easier to handle.

Due to the violent motion of the intracluster plasma during cluster collapse, the final magnetic field strength depends on the dynamics of the cluster formation. Starting with identical initial magnetic field set-ups, final field strengths differ by a factor of 4-5, depending essentially 

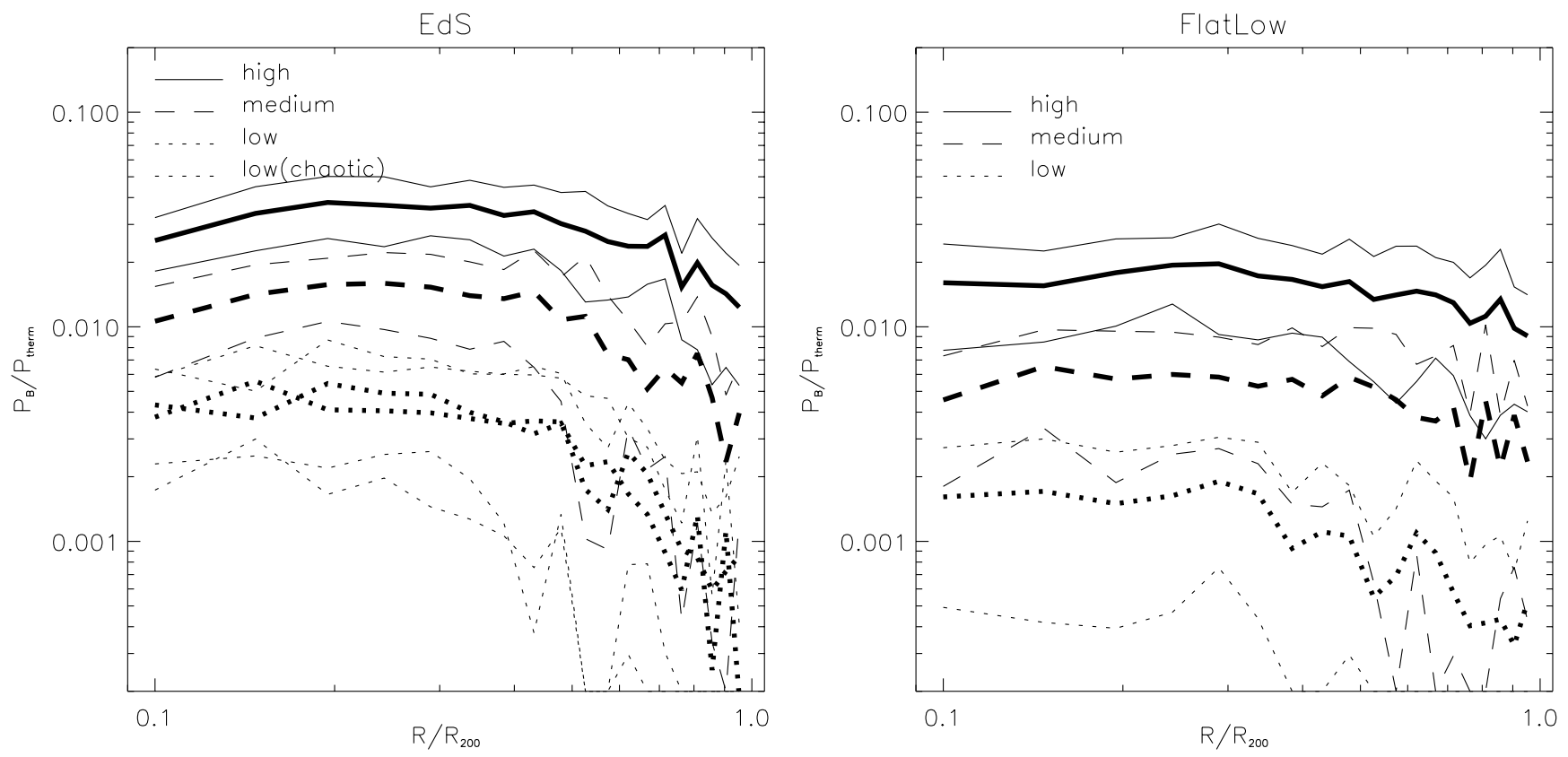

Fig. 1. Shown here is the ratio between the magnetic and the thermal pressure in shells around the cluster centre as a function of radius in units of the virial radius $r_{200}$ (heavy lines). Different line types distinguish the different initial magnetic field strengths as indicated in the plot. The two dotted lines represent the low field with homogeneous and with chaotic initial field configurations. Both lead to the same amount of pressure support in the final clusters. The curves show the average across the entire simulated cluster sample. The thin lines indicate the $r m s$ scatter within the sample. Near cluster centres, the ratio between magnetic and thermal pressure is consistent with the isotropic estimate of Eq. (1). The left panel represents the realisations for the EdS cosmology, the right panel for the FlatLow cosmology

on cluster mass. The mean magnetic field strength drops by about two orders of magnitude between the cluster centre and the virial radius. A detailed study of the evolution and the structure of intracluster magnetic fields in different cosmologies will be described in a forthcoming paper.

A direct way to compare simulated magnetic fields in galaxy clusters with observations is provided by Faradayrotation measurements. We created samples of synthetic rotation measures by integrating the Faraday rotation along $10^{4}$ light rays randomly shot through each of a sub-set of our cluster simulations. As in DBL99, the cumulative rotation-measure distributions obtained in simulations with either homogeneous or chaotic initial field set-up were statistically identical, confirming once again that the structure of the initial field has no observable consequences for the structure of the final field processed by cluster collapse.

We further performed two types of comparison between synthetic and observed Faraday-rotation data. In the first, we compared the radial distribution of rotation measures seen in the Coma cluster (Kim et al. 1990) with that obtained from one of our simulated clusters with mass similar to Coma. In the second, the sample of rotation measures compiled by Kim et al. (1991) from observations of many Abell clusters was compared to synthetic rotation measures obtained from our set of simulated clusters. In both cases, synthetic rotation-measure distributions were obtained along $10^{4}$ light rays randomly shot through the simulations.

Both comparisons showed that the medium initial magnetic field strength could reproduce the observed statistics very well. In both cases, the simulated and the observed rotation-measure distributions could not significantly be distinguished in a Kolmogorov-Smirnov test. However, the scatter in both measurements and simulations is large. This is due to the relatively low number of simulated clusters per set, and to the selection criteria of galaxy clusters involved in the simulations and the observations. We therefore decided to allow for a range of initial magnetic field strengths which results in a range of synthetic Faraday-rotation distributions which are still well in agreement with the observed data. Further detail can be found in DBL99 and Dolag (2000). Comparing with more recent data by Clarke et al. (1999), we find identical or even improved agreement. A study of clusters in different cosmologies, using newer observational data, is under way.

Choosing a realistic model to distribute relativistic electrons in the simulated clusters, the simulations are also able to match the properties of observed radio-haloes very well, including the observed very steep relation between cluster temperature and radio luminosity (see Dolag \& Ensslin 2000). 

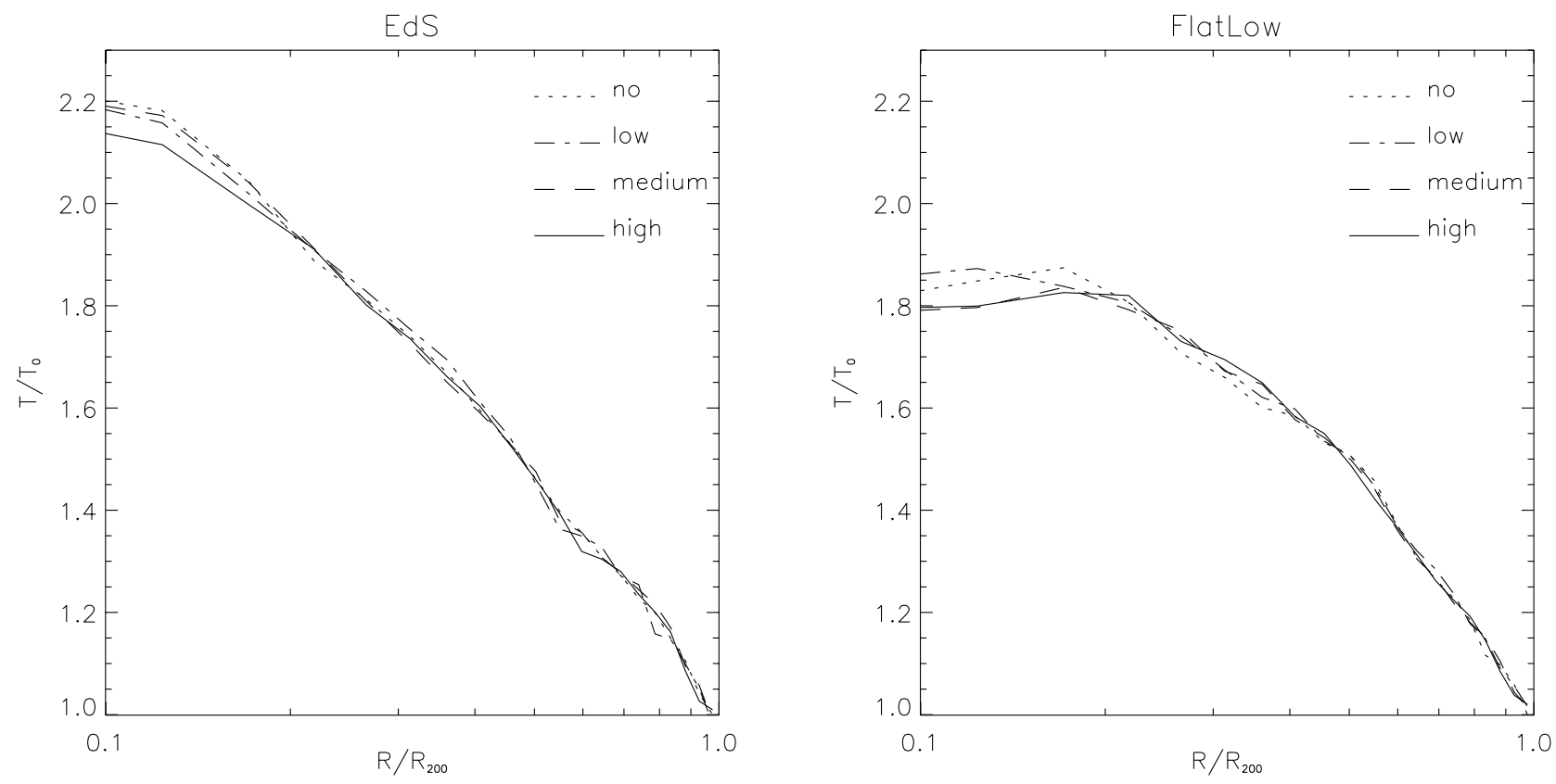

Fig. 2. This figure shows the radial temperature profile, averaged across the simulated cluster sample. The temperature is scaled by the mass-weighted temperature of the non-magnetised cluster at a radius of $r_{200}$, and the radius is scaled by $r_{200}$. Different line types distinguish different initial magnetic field strengths, as indicated in the plot. The temperature change with increasing magnetic field strength is clearly visible. The temperature decrease near cluster centres is compatible with the isotropic estimate of Eq. (1). As in Fig. 1, the left panel represents the EdS cosmology, the right panel the FlatLow cosmology

\subsection{Quantities calculated}

The correlation length of final magnetic field configurations near cluster centres is of order $50 \mathrm{kpc}$ (see DBL99). Field orientations in regions large compared to the correlation length can therefore be considered as randomised. For instance, there is no preferred direction in the final magnetic pressure distribution. Thus, we can safely average thermodynamic quantities like the gas pressure and gas temperature in spherical shells, provided these shells are sufficiently thick compared to the field correlation length.

The radial distances to the cluster centres are scaled by the virial radius $r_{200}$. For simulated clusters, $r_{200}$ is defined as the radius enclosing a mean density $\bar{\rho}\left(r_{200}\right)$ of 200 times the critical density $\rho_{\text {cr }}$,

$\bar{\rho}\left(r_{200}\right)=\frac{3 M\left(r_{200}\right)}{4 \pi r_{200}^{3}}=200 \rho_{\text {cr }}$.

The temperature profiles give mass-weighted temperatures averaged in shells, divided by the mass-weighted temperatures in the shell at the radius $r_{200}$ of the corresponding non-magnetised cluster.

\section{Results}

During cluster formation and evolution, the simulated intracluster magnetic field strengths are amplified by compression, tangling and bending of field lines, and shear flows in the gas. The magnetic field structure is also affected by merging, accretion of sub-clumps, and consequent relaxation processes. In combination, these processes amplify the initial magnetic fields in the simulation by factors of order $\approx 1000$. Final fields reach strengths of a few $\mu \mathrm{G}$ in and near cluster centres, in good agreement with field strengths inferred from observations. The growing magnetic fields affect the balance between the gravitational force and the total (magnetic plus thermal) pressure during cluster formation.

\subsection{Radial structure}

On the whole, the fairly isotropic magnetic pressure has the tendency to counteract the gas collapse, and therefore acts in a similar manner as the thermal pressure. This implies that the gas stratification can achieve hydrostatic equilibrium at lower temperature, because lower thermal pressure is required in presence of the additional pressure support due to the magnetic field. Figure 1 shows, averaged in shells, the ratio

$\mathcal{F}_{\mathrm{p}}(R)=\frac{p_{\mathrm{B}}(R)}{p_{\mathrm{th}}(R)}=\frac{\left\langle|\boldsymbol{B}|^{2} / 8 \pi\right\rangle_{R_{1} \leq R \leq R_{2}}}{\left\langle p_{\mathrm{th}}\right\rangle_{R_{1} \leq R \leq R_{2}}}$

between the magnetic pressure $p_{\mathrm{B}}$ and the thermal pressure $p_{\text {th }}$ as a function of the radius $R$. Due to compression and turbulence in the gas flow, the magnetic field is highest in and near the centres of the simulated clusters, where the thermal pressure is also highest. As Fig. 1 shows, the relative contribution of magnetic fields to the pressure support appears to peak somewhere between the cluster core radii and the virial radii $r_{200}$. This feature, however, is not very pronounced, so that the ratio between magnetic 

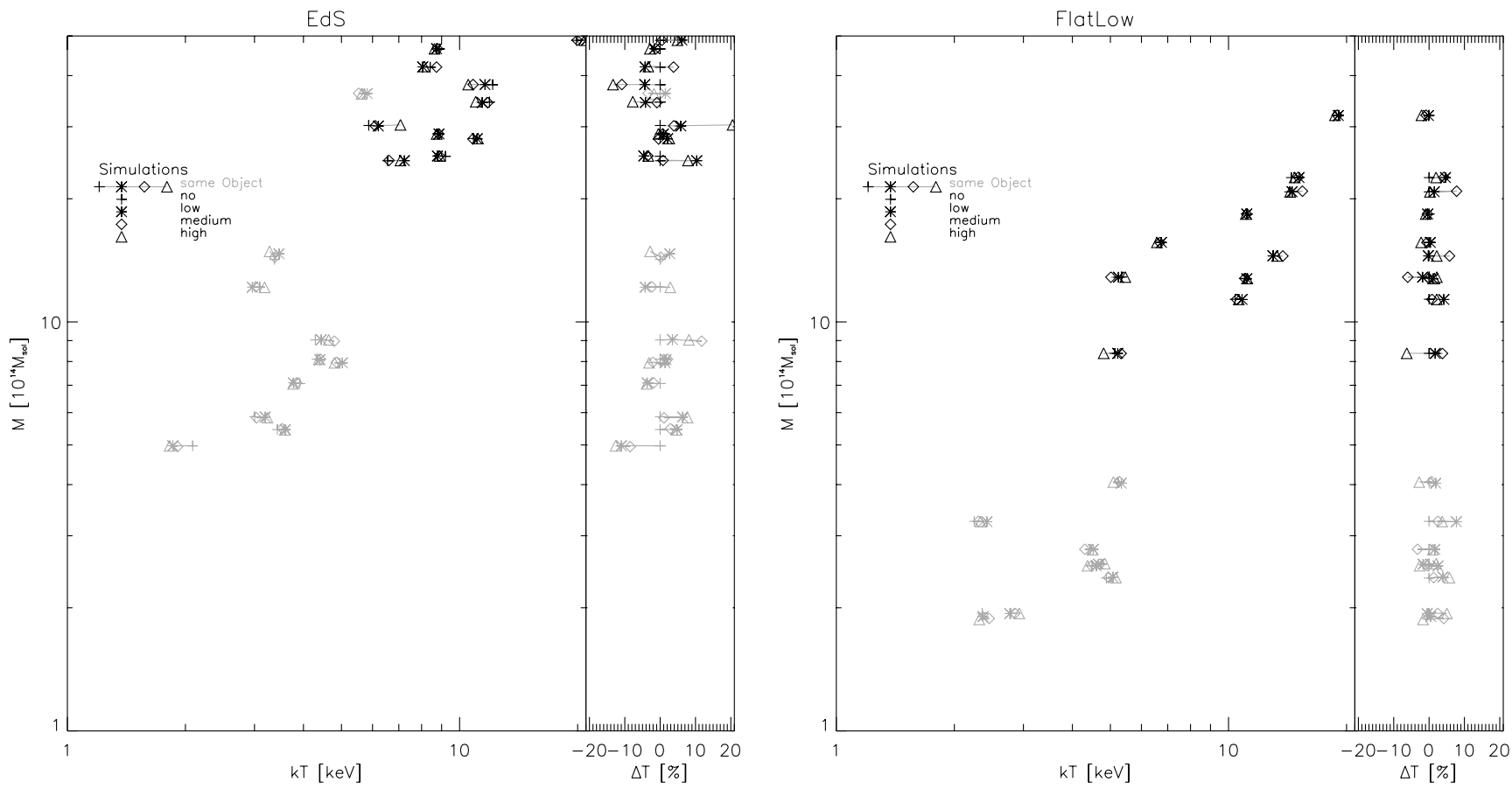

Fig. 3. The large panels show the temperature-mass relation for the simulated cluster sample. Here, the temperature is the emission-weighted temperature within the radius $r_{200}$. All clusters are simulated with different initial magnetic field strengths marked by different symbols, as indicated in the plot. The right-hand overlays show the temperature change relative to nonmagnetised clusters for the different initial magnetic field strengths in percent for each simulated cluster. The large scatter is obvious, but there is a general tendency towards lower temperatures in magnetised clusters. To follow this relation towards lower temperatures, smaller objects (marked as gray symbols) found in the simulations are also used, which lead to a total of 20 different clusters for the EdS cosmology (left panel) and 18 different clusters for the FlatLow cosmology (right panel). As low mass objects are represented by less particles, the resolution in these objects drops towards lower masses and the scatter in these objects is expected to be larger

and thermal pressure support is almost constant across a large range of cluster-centric radii. Due to the smaller final masses of the clusters in the FlatLow cosmology, the magnetic pressure support is also smaller in these clusters. Nevertheless, these less magnetized clusters are able to match the observed Faraday-rotation. This is because the value of $H_{0}$ is chosen higher in the low-density universe, so that the projected, physical distances of the measurement locations with respect to the cluster centre shrink. Closer to the cluster centre, the magnetic field is generally higher, and therefore these less magnetised clusters well reproduce the measurements.

The overall structure of the magnetic field in the outer regions of the clusters differs from that near the cluster centre. Accretion and merger events arrange the magnetic fields in the outer cluster regions in coherent patterns on fairly large scales. Near the cluster centre, the gas flow pattern is almost randomised, and the magnetic field is consequently tangled and bent on fairly small scales. Although the magnetic pressure can be considered isotropic at or near the cluster centre, it may well be anisotropic further out. Even though the fraction of the magnetic relative to the thermal pressure, averaged within spherical shells, is approximately constant across the cluster, the detailed effects of magnetic pressure on the gas flow depend on the direction of the magnetic field relative to other locally preferred directions, like the orientation of filaments surrounding a cluster, the local path of matter infall, the orbit of a merging sub-clump and such. Therefore, the same overall magnetic pressure can have different effects on the local dynamics of gas flows, depending on whether the magnetic field is ordered on scales comparable to the cluster scale, and on the orientation of the magnetic field. In addition, the magnetic field alters the dynamical time scale of a (simulated) cluster. Therefore, when comparing simulated clusters with and without magnetic fields at equal redshifts, we must be aware that we may be comparing them at slightly different evolutionary stages according to their intrinsic timescale.

Figure 2 shows the temperature profile averaged across the set of ten simulated clusters. On the whole, the temperature increases towards the cluster centre by a factor of $\approx 2$. For different cosmologies, the final clusters have a different amount of substructure. This leads to different shapes of the temperature profiles for the clusters in the two different cosmologies used. Although the detailed temperature profile of an individual cluster depends on its dynamical state and formation history, the tendency is clearly visible that the cluster temperature decreases with increasing initial magnetic field strength towards the cluster centre. Deviations from this general trend are possible in individual clusters because of the reasons mentioned 
before. The mean across the entire cluster sample, however, reveals a monotonic trend increasing towards the cluster centre. This behaviour is expected in view of the previous discussion about the impact of the magnetic field in different parts of the cluster.

\subsection{Temperature-mass relation}

The effect of magnetic fields on the temperature-mass relation in galaxy clusters is shown in Fig. 3. Different initial magnetic field strengths are marked with different symbols. As shown before, the effect of the magnetic pressure on the temperature is on average strongest near cluster centres. Therefore, the strength of the effect on the temperature-mass relation depends on the chosen radius within which the mean temperature is calculated. It changes also if we choose the emission-weighted instead of the mass-weighted temperature. For Fig. 3, we have chosen to calculate the emission-weighted temperature within the virial radius $r_{200}$ in order to be comparable to the measurements. We also translated the masses of the simulated galaxy clusters to velocity dispersions. Figure 3 also shows the measured relationship between cluster temperatures and velocity dispersion as dashed-dotted line. The fairly large scatter in individual clusters is clearly seen. In the mean, the temperature difference between the magnetised and non-magnetised clusters is $\approx 5 \%$, and it can go up to $10-15 \%$ in individual clusters.

Table 3. This table shows the temperature difference between the magnetised and non-magnetised clusters (Cols. 2 and 4), and the rms (Cols. 3 and 5) of this distribution, for the three different initial magnetic field strengths. Columns 2 and 3 are for the EdS, Cols. 4 and 5 for the FlatLow cosmologies, respectively. The temperatures are emission-weighted temperatures within the virial radius $r_{200}$. All clusters shown in Fig. 1 are used

\begin{tabular}{|l||c|c||c|c|}
\hline model & $\langle|\Delta T|\rangle_{\text {EdS }}$ & rms & $\langle|\Delta T|\rangle_{\text {FlatLow }}$ & rms \\
\hline low & $4.3 \%$ & $2.7 \%$ & $2.0 \%$ & $2.1 \%$ \\
medium & $3.1 \%$ & $3.7 \%$ & $2.4 \%$ & $2.1 \%$ \\
high & $5.8 \%$ & $5.1 \%$ & $2.5 \%$ & $1.7 \%$ \\
\hline
\end{tabular}

Although the systematic effect of magnetic fields on the emission-weighted temperature profiles is small, the magnetic fields also increase the scatter in the masstemperature relation. Values for the rms scatter are given in Table 3. This effect is more pronounced in the EdS cosmology because of the smaller gas fraction in a highdensity universe.

\section{Discussion}

We tested the influence of the non-thermal pressure support due to magnetic fields in galaxy clusters. To do so, we performed cosmological MHD simulations of ten clusters in two different cosmologies, each with four different initial magnetic field configurations, thus yielding a total of 90 cluster models.

We chose initial magnetic field strengths such that the final intracluster fields were able to statistically reproduce the amplitude and spatial distribution of observed Faraday-rotation measurements. For such fields, we found a relatively small non-thermal pressure support of about $5 \%$. The temperature change in the cluster cores is therefore on average $\approx 5 \%$, with the general tendency towards decreasing temperature with increasing magnetic field strength. This tendency is most pronounced in cosmologies with a low gas fraction, i.e. a high dark-matter density. The effect of the magnetic fields can be substantially stronger in individual clusters, introducing a cosmology-dependent scatter of up to (10-15)\% into the mass-temperature relation.

Our overall conclusion is that the statistical rotationmeasure data, taken in the most straightforward interpretation, suggests that magnetic fields are generally dynamically unimportant in galaxy clusters. We are aware that simulations like those presented here do not include the full range of physical processes required for a definitive calculation of intracluster fields, like small-scale turbulence and non-ideal magneto-hydrodynamics. A detailed study of such processes is currently out of reach for realistic numerical simulations, rendering our simulations illustrative rather than definitive. We point out, however, that the inclusion of magnetic fields in cluster simulations does constitute an improvement over previous studies, even if it confirms earlier conjectures.

Acknowledgements. We wish to thank Harald Lesch for useful discussions, and the referee, Jean Eilek, for her very detailed and thoughtful report.

\section{References}

Brio, M., \& Wo, C. C. 1988, J. Comput. Phys., 75, 400

Clarke, T. E., Kronberg, P. P., \& Böhringer, H. 1999, Proceedings of "Diffuse Thermal and Relativistic Plasma in Galaxy Clusters", ed. H. Böhringer, L. Feretti, \& P. Schuecker, MPE Report 271, 82, 1999

Dolag, K., Bartelmann, M., \& Lesch, H. 1999, A\&A, 348, 351

Dolag, K. 2000, Proceedings of "Constructing the Universe with Clusters of Galaxies", IAP 2000, ed. F. Durret, \& D. Gerbal [astro-ph/0010149]

Dolag, K., \& Ensslin, T. 2000, A\&A, in press [astro-ph/0010296]

Kim, K. T., Kronberg, P. P., Tribble, P. C. 1991, ApJ, 379, 80

Kronberg, P. P. 1994, Rep. Prog. Phys., 57, 325

Lucy, L. 1977, AJ, 82, 1013

Monaghan, J. J. 1992, ARA\&A, 30, 543

Steinmetz, M. 1996, MNRAS, 278, 1005

Sugimoto, D., Chikada, Y., Makino, J., Ito, T., Ebisuzaki, T., \& Umemura, M. 1990, Nature, 345, 33

Vallee, J. P., MacLeod, J. M., \& Broten, N. W. 1986, A\&A, 156,386

Vallee, J. P., MacLeod, J. M., \& Broten, N. W. 1987, ApL, 25, 181

Wu, X., Fang, L., \& Xu, W. 1998, A\&A, 338, 813 